\title{
Chronic oscillating glucose challenges disarrange innate immune homeostasis to potentiate the variation of neutrophil-lymphocyte ratio in rats with or without hidden diabetes mellitus
}

This article was published in the following Dove Press journal:

Diabetes, Metabolic Syndrome and Obesity:Targets and Therapy

\begin{abstract}
Gaoxiong Yang, ${ }^{*}$ Rui Yan, ${ }^{2 *}$ Huanjun Tong, ${ }^{3 *}$ Jitai Zhang,' Bin Chen, ${ }^{4}$ Xiangyang Xue, ${ }^{5}$ Jue Wang, ${ }^{6}$ Maoping Chu, ${ }^{7}$ Shengwei Jin, ${ }^{8}$ Ming $\mathrm{Li}^{\prime}$

'Cardiac Regeneration Research Institute, Wenzhou Medical University, Wenzhou, China; ${ }^{2}$ School of Laboratory Medicine and Life Science, Wenzhou Medical University, Wenzhou, China; ${ }^{3}$ The Second Clinical Medical College, Wenzhou Medica University, Wenzhou, China; ${ }^{4}$ Department of Medical Ultrasound, The First Affiliated Hospital of Wenzhou Medical University, Wenzhou, China; ${ }^{5}$ Department of Microbiology and Immunology, Institute of Molecular Virology and Immunology, Institute of Tropical Medicine, Wenzhou Medical University, Wenzhou, China; ${ }^{6}$ Department of Cardiac Surgery, The First Affiliated Hospital of Wenzhou Medical University, Wenzhou, China; ${ }^{7}$ Children's Heart Center, The Second Affiliated Hospital and Yuying Children's Hospital, Institute of Cardiovascular Development and Translational Medicine, Wenzhou Medical University, Wenzhou, China; ${ }^{8}$ Department of Anesthesia and Critical Care, The Second Affiliated Hospital of Wenzhou Medical University, Wenzhou, China

*These authors contributed equally to this work
\end{abstract}

Correspondence: Ming Li

Cardiac Regeneration Research Institute,

Wenzhou Medical University, Chashan Higher Education Park, Wenzhou, 325035 Zhejiang,

China

Email mingli@wmu.edu.cn

Shengwei Jin

Department of Anesthesia and Critical Care, The Second Affiliated Hospital of Wenzhou Medical University, Chashan Higher Education Park, Wenzhou, 325035 Zhejiang, China Email jinshengwei69@I63.com
Background: The neutrophil-lymphocyte ratio (NLR) has been considered as an inflammatory marker in various disorders, but it is not clear whether the NLR is also elevated with hidden diabetes (HD), which is normal in fasting blood glucose (FBG) but abnormal in the oral glucose tolerance test (OGTT).

Materials and methods: An HD animal model for 27 days and an animal model with oscillating glucose (OG) for 7 days were applied on adult female Sprague-Dawley rats. OGTT, leukogram analysis, histology, and immunohistochemistry were carried out.

Results: In HD rats, the percentage of neutrophils increased but the percentage of lymphocytes decreased; hence, the NLR rose relative to sham. This may be a result of the OG levels often experienced by diabetic subjects, as normal rats given OG $(6 \mathrm{~g} / \mathrm{kg} / 6 \mathrm{~h})$ for 7 days had significantly reduced lymphocyte numbers and increased NLR compared with the values before and $1 \mathrm{~h}$ after oral glucose administration during OGTT. Glucose-induced disarrangement of partitions of circulating immune cells and NLR was involved in the increase in oxidative stress, as these changes were totally blocked by the antioxidant glutathione (GSH). GSH ( $50 \mathrm{mg} / \mathrm{kg} / 6 \mathrm{~h}$ ) totally blocked the glucose-induced alterations in lymphocyte and NLR values.

Conclusion: HD associated with elevation of NLR values may be partly attributed to a homeostasis disorder of the innate inflammatory state, caused by oscillating hyperglycemia. Acute high glucose administration produced a significant decrease in lymphocyte number. OG administration potentiated this effect and increased the NLR value, which was blocked by GSH, suggesting that reactive oxygen species play a critical role in maintaining lymphocyte numbers. Keywords: neutrophil-lymphocyte ratio, diabetes, oscillating glucose level, innate immune, rat

\section{Introduction}

The neutrophil-lymphocyte ratio (NLR) is considered to be a marker of low-grade inflammation in diabetes mellitus (DM). ${ }^{1,2}$ Research demonstrates that neutrophils, the first cells to respond to inflammation, can aggravate the inflammatory state by interacting with macrophages and antigen-presenting cells to induce type 1 diabetes (T1D). ${ }^{3}$ A high NLR represents leukogram shifts to either a higher number of neutrophils or a lower number of lymphocytes, or both, from the basal innate immune homeostasis. However, the pathological development of NLR in DM remains unclear. The literature to date is contradictory in regard to the changes in neutrophil numbers in patients with DM. The levels of circulating neutrophils have been reported to be increased ${ }^{4,5}$ or decreased ${ }^{6,7}$ in patients with T1D. The disagreement in alterations of neutrophils 
was thought to be due to different studies coming from the discoveries of different stages of the pathological progress of DM, or from multiple unrecognized factors (mild infections and mild chronic disorders associated with inflammation, etc.) that could alter the NLR in clinical studies, or simply that neutrophils may not be the main cause of the increased NLR.

The development and regulation of the homeostasis of the innate immune system is dependent on the daily suicide of lymphocyte clones and, hence, cell death by apoptosis plays a pivotal role in purging the body of dangerous lymphocytes. ${ }^{8}$ T1D is an autoimmune disorder which may be related to defects in lymphocyte apoptosis in this disease. ${ }^{9}$ In 1948, Jailer et al found that oral glucose challenge in both diabetics and nondiabetics produced a significant decrease in circulating lymphocytes, which was much greater in diabetic patients. ${ }^{10}$ This phenomenon has been confirmed by later studies in which the rate of lymphocyte apoptosis was significantly higher in type 2 diabetic patients than that in the healthy population. ${ }^{11}$ These findings suggest that the increase in NLR value may simply result from the decrease in lymphocyte number, which is caused by high-glucose-induced oxidative stress and cell apoptosis.

A diabetic animal model could be applied to test this hypothesis. Compared to clinical studies, experimental animal models have the advantage of using controlled experimental designs to systematically assess the diagnostic value of NLR during the pathological development of T1D induced by alloxan. Alloxan (2,4,5,6-tetraoxypyrimidine; 2,4,5,6-pyrimidinetetrone) is a derivative of pyrimidine used to selectively destroy pancreatic $\beta$-cells. ${ }^{12,13}$ In the alloxan-induced diabetes animal model, there a substantial number of rats failed to increase their fasting blood glucose (FBG) level. Alloxan is not commonly used to establish the diabetic model as the percentage incidence of diabetes is quite variable. Alloxan produced only $72 \%$ ofT1D rats with abnormal FBG. ${ }^{14}$ Female animals have been reported to be more resistant than males to the chemically induced $\beta$-cell cytotoxicity. ${ }^{15}$ Therefore, we chose alloxan-treated female rats with normal FBG but with an abnormal oral glucose tolerance test (OGTT) as a hidden diabetes (HD) model. In the HD model, we determined the dynamic alteration of neutrophil and lymphocyte counts, NLR, and renal damage, to show that NLR together with OGTT can be used as an indicative marker and diagnostic reference for HD. To determine the cause of the increase in NLR in HD animals, whether it results from diabetic inflammation or the oscillating glucose (OG)-induced disorder of innate immune homeostasis which is often experienced in diabetic subjects, we administered oral OG $(6 \mathrm{~g} / \mathrm{kg} / 6 \mathrm{~h})$ to normal rats for 7 days and examined the leukogram pattern before and $1 \mathrm{~h}$ after oral glucose administration during the OGTT period.

\section{Materials and methods}

Ninety-three Sprague-Dawley female rats with a body weight of $200 \pm 10 \mathrm{~g}$ (mean $\pm \mathrm{SD}$ ) were purchased from the Chinese Academy of Medical Sciences (Shanghai, China). Rats were acclimatized under a controlled environment at $22 \pm 1^{\circ} \mathrm{C}$ and a $12 \mathrm{~h}$ light/dark cycle, with free access to food and water, for 2 weeks prior to the experiments. The research protocol was approved by the Institutional Animal Care and Use Committee of Wenzhou Medical University, China. All experiments were conducted according to the guidelines of the committee.

\section{Induction and management of HD}

Rats were divided into sham $(n=14)$ and alloxan-induced HD $(n=12)$ groups. HD was induced by 2 intraperitoneal injections of alloxan, $100 \mathrm{mg} / \mathrm{kg}$ body weight on 2 sequential days. The criterion for inclusion in the HD group was an FBG $\leq 110 \mathrm{mg} / \mathrm{dL}$ but abnormal OGTT during the experimental period. Rats were weighed daily throughout the study and the body weights were recorded. The changes in body weight were calculated as the differences in body weight between postalloxan treatment day and prealloxan treatment day (P0). Before FBG measurements, the rats were fasted for $12 \mathrm{~h}$ (22:00-10:00 h). FBG was measured with an Accu-Chek ${ }^{\circledR}$ reflectance meter with Accu-Chek active test strips (Roche Diabetes Care, Mannheim, Germany) on P0, and postalloxan treatment days P5, P7, P16, and P27. During experimental days P24-P25, rats were maintained in metabolic cages for $24 \mathrm{~h}$ to collect urine. The water intake, food intake, and urine volume were measured and recorded. The urine aliquots were stored frozen for the subsequent measurement of albumin by enzyme-linked immunosorbent assay (Rat Albumin ELISA Kit, AssayMax ${ }^{\mathrm{TM}}$; Assaypro, St. Charles, MO, USA).

\section{Animal model with $O G$ administration}

Animals were given either $6 \mathrm{~g} / \mathrm{kg}$ glucose or distilled water ( $2 \mathrm{~mL} / 100 \mathrm{~g}$ body weight, $\mathrm{n}=7$ for both groups) by gavage every $6 \mathrm{~h}$ (at 06:20, 12:20, 18:20, and 24:20 h) for 7 days. To test the hypothesis that a disorder of innate immune homeostasis is induced by OG-produced oxidative stress, an antioxidant, glutathione (GSH, $50 \mathrm{mg} / \mathrm{kg} / 6 \mathrm{~h}, \mathrm{n}=7$ ), was intraperitoneally injected into animals with OG challenge as described as above. For leukogram analysis, the tail blood was collected preadministration and $1 \mathrm{~h}$ post- $2 \mathrm{~g} / \mathrm{kg}$ glucose administration on treatment day 7 .

\section{OGTT}

On experimental days P7 and P27, the OGTT was performed on overnight-fasted (22:00-10:00 h) normal and alloxantreated rats. A glucose load ( $2 \mathrm{~g} / \mathrm{kg}$ body weight) was given 
to each rat orally with a feeding syringe. Blood samples were collected from the tail vein at $0,30,60,90$, and $120 \mathrm{~min}$ of glucose administration, and the blood glucose level was determined using a glucometer (Accu-Chek Active; Roche, Mannheim, Germany).

\section{Leukogram analysis}

To obtain repeated measures in the same animal, blood samples were taken from the tail of each animal at P7 and P27 in both sham and HD groups. To examine the effects of hyperglycemia in the leukogram, blood samples were also collected on P27 in the diabetic group (DM group, FBG: mean \pm SD $227.10 \pm 119.96 \mathrm{mg} / \mathrm{dL}, \mathrm{n}=6$ ). The identification and differential counting of leukocytes were performed with samples stained by Wright's dye under an optical microscope. As previous studies reported that some lymphocytes and neutrophils were difficult to differentiate and recognize by machine and some neutrophils were counted as monocytes by the LaserCyte, ${ }^{16,17}$ to determine the leukocyte classifications more accurately, manual differential counts were performed by 2 technicians in a blinded manner. The NLR was calculated by dividing the neutrophil count by the lymphocyte count.

\section{Histology}

To allow the kidney to recover from the acute mildly nephrotoxic effects of toxic chemicals over more than 3 weeks, ${ }^{18}$ studies examining the effects of treatments on the development of diabetic nephropathy were executed 27 days after alloxan treatment. The rats were killed and tissue samples were taken from the pancreas and kidney for histological studies. The samples were fixed in $2 \%$ buffered paraformaldehyde and embedded in paraffin. Then, $5 \mu \mathrm{m}$ slices were made from the paraffin blocks which were stained with Picro Sirius red with or without hematoxylin as counterstaining. In brief, sections were stained in Sirius red solution for $1 \mathrm{~h}$, washed in acetic acid, counterstained with hematoxylin, quickly dehydrated, and then mounted in a resinous medium. The stained tissue sections were scanned using a Leica fluorescence microscope (Leica DM6000B; Leica, Wetzlar, Germany) and Leica LAS X software. The sample was quantified using image analysis software (ImageJ; NIH, Bethesda, MD, USA).

The glomerular volume was quantified as described previously. ${ }^{19}$ The method needs only determination of the mean glomerular random cross-sectional area, $A(G)$. The mean glomerular volume is obtained as:

$$
V(G)=\beta / K \times[A(G)]^{3 / 2}
$$

where $\beta=1.38$ pertains to spheres, and $K$ (a distribution coefficient) is taken to be 1.10 . The counting was done in an unbiased counting frame on systematically sampled visual fields at $400 \times$ magnification using a projection head fitted to the microscope. In each animal, 812-1076 glomerular profiles, which were defined as the minimal convex polygon circumscribing the capillary tuft, were analyzed. The histological parameters were quantified by two independent operators in a blinded manner.

\section{Immunohistochemistry}

On experimental days P7 and P27, pancreas samples were fixed in $2 \% \mathrm{w} / \mathrm{v}$ paraformaldehyde for $2 \mathrm{~h}$ and processed for immunohistochemistry as described previously. ${ }^{20}$ After preblocking, pancreas samples were stained with mouse anti-insulin (66198-1-Ig, Proteintech, 1:500) and rabbit anti-glucagon (PB0742, Boster, 1:200) antibodies in 5\% v/v goat serum. All secondary antibodies (Alexa Fluor 488 or 594 goat anti-mouse or anti-rabbit) were from Molecular Probes, Waltham, MA, USA. DAPI was used to stain nuclei. Whole-sample images were scanned and assembled on a Leica fluorescence microscope (Leica DM6000B) using Leica LAS X software. For quantitative immunohistochemistry, the relative expression/ $\mathrm{mm}^{2}$ determined from 200 fields $(40 \times)$ of pancreas from each rat was quantified using ImageJ software. The average $\beta$-cell and $\alpha$-cell number and their ratio per islet of pancreas were quantified by two independent operators in a blinded fashion.

\section{Statistics}

Results are presented as the mean \pm SD. Statistical significance was determined by Student's $t$-test or using analysis of variance (ANOVA) with Tukey tests for posthoc comparisons at a $1 \%$ or $5 \%$ significance level of difference. The relationships between variables were determined by linear regression analysis. GraphPad Prism 6.0 software (La Jolla, CA, USA) was used to calculate the OGTT area under the curve (AUC) and for statistical analyses.

\section{Results}

\section{Animal models}

Rats injected with two sequential $100 \mathrm{mg} / \mathrm{kg}$ doses of alloxan produced two cohorts of animals. The first group (81\%) developed a gradually increasing hyperglycemia from day 7 onward. In contrast, the FBG levels measured on experimental days P5, P7, P16, and P27 were normal (74-110 mg/dL) in the second alloxan-treated cohort (Figure 1A). Alloxantreated rats with normal FBG but abnormal OGTT were considered as successful HD animals. On treatment day P7, 
A

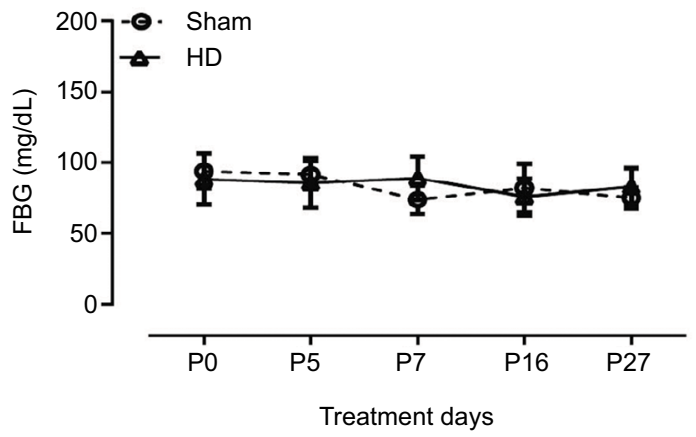

C

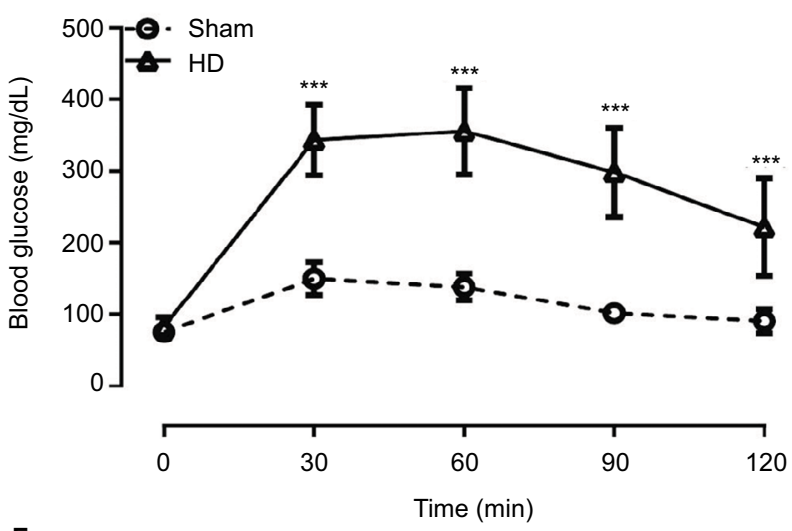

B

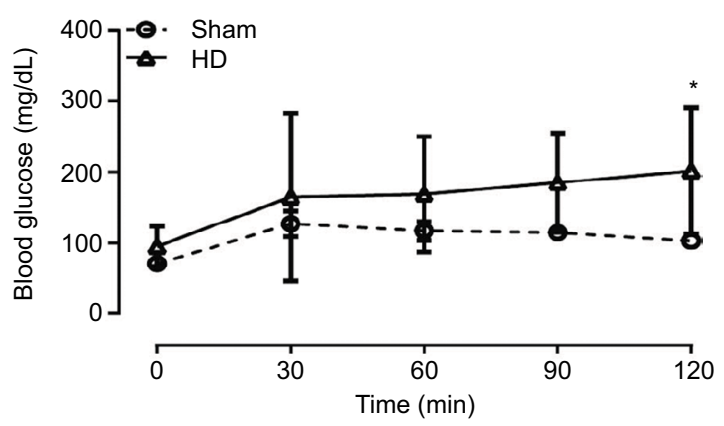

D
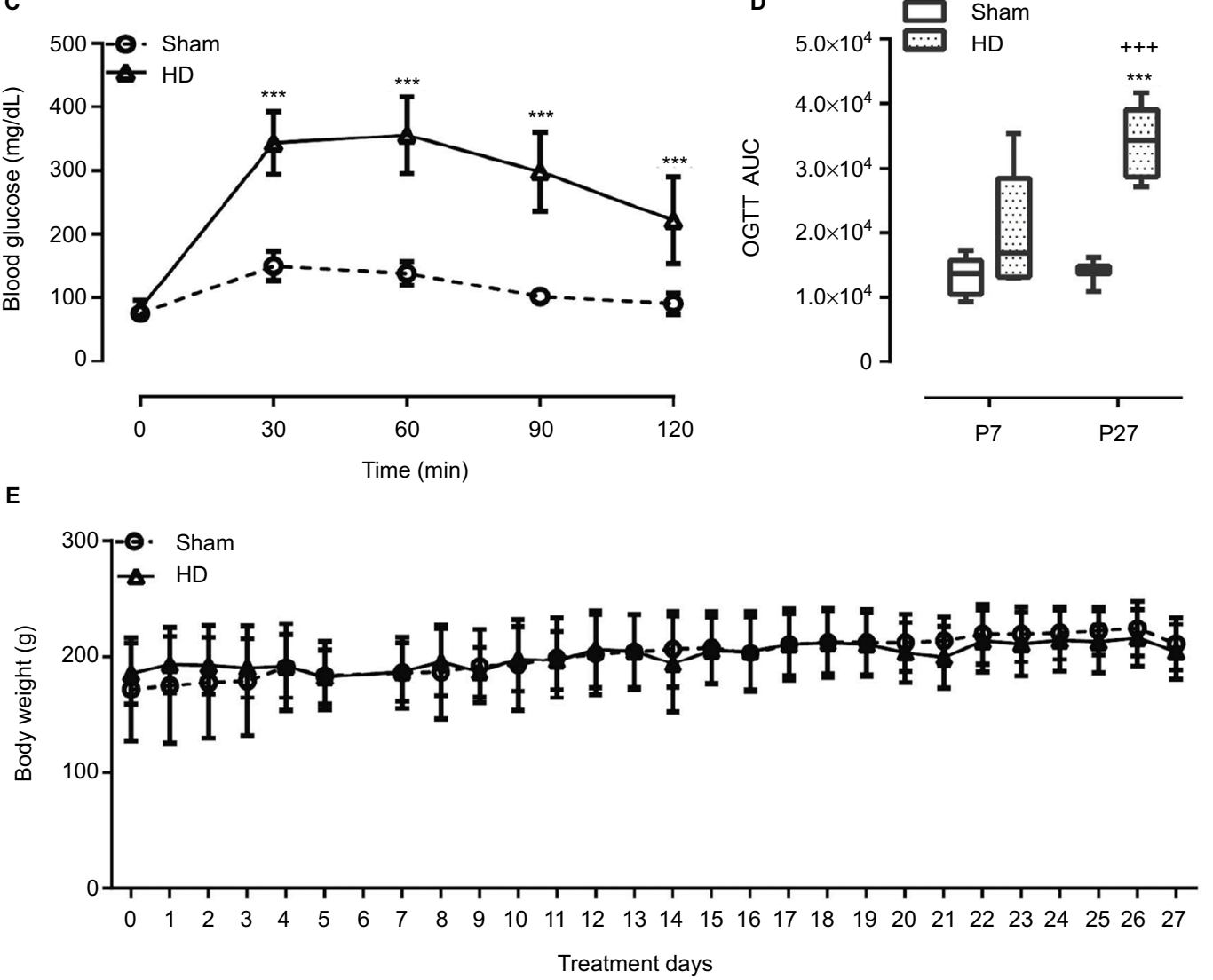

F

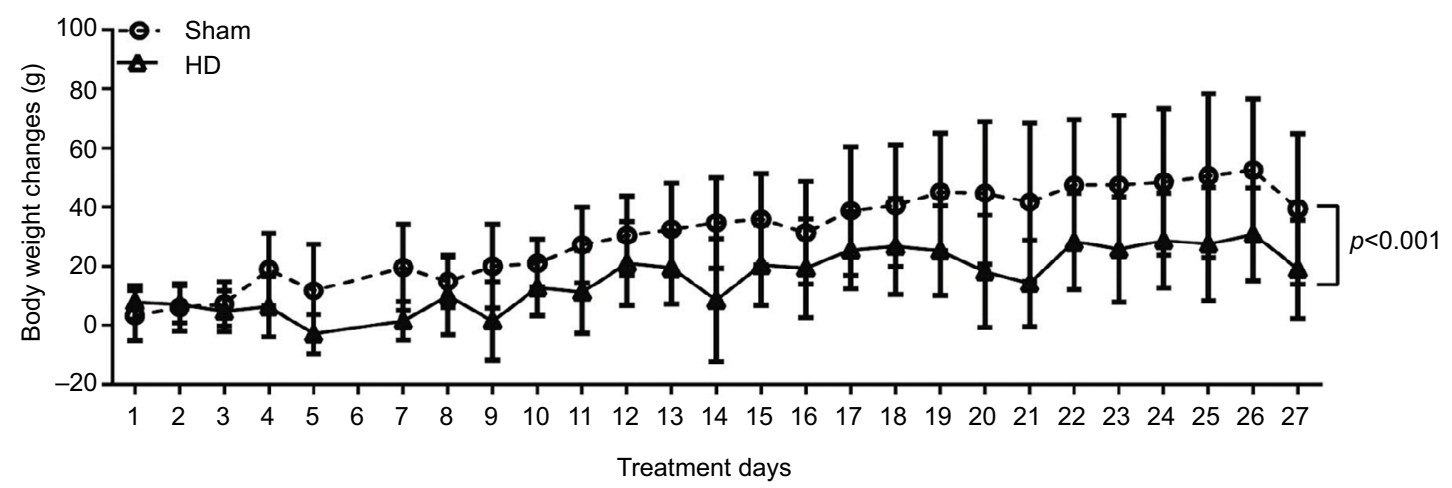

Figure I Alloxan produced the development of $H D$ in female rats (sham $n=8, H D n=7$ ).

Notes: (A) FBG levels during experimental period P0-P27. (B) OGTT after 7 days of alloxan treatment. (C) OGTT after 27 days of alloxan treatment. (D) OGTT AUC after 7 and 27 days of alloxan treatment. (E) Body weights were measured daily during experimental period P0-P27. (F) Body weight changes during experimental period PI-P27. The changes were obtained from the difference in body weight between posttreatment day PI-P27 and pretreatment day P0. The data for OGTT AUC were analyzed by unpaired Student's $t$-test. The data for FBG, OGTT, body weight, and body weight changes were analyzed by 2-way analysis of variance with Tukey tests for posthoc comparisons. ${ }^{*} p<0.05$ and $*^{* * *} p<0.001$ sham vs HD; ${ }^{+++} p<0.001$ P7 HD vs P27 HD.

Abbreviations: HD, hidden diabetes; FBG, fasting blood glucose; OGTT, oral glucose tolerance test; AUC, area under the curve; P0, prealloxan treatment day; PI, posttreatment day I; P7, posttreatment day 7; P27, posttreatment day 27. 
the OGTT glucose levels after 120 min glucose administration were significantly higher in HD than in sham rats (Figure 1B). The OGTT glucose levels at multiple time points were further increased in HD animals on P27 (Figure 1C). The OGTT AUC value on P27 was also significantly higher than that on P7, as expected (Figure 1D). During the experimental period, there were no differences in body weight between sham and HD animals (Figure 1E). However, relative to sham, animals rendered HD by alloxan injection exhibited a significant reduction in body weight gain (Figure 1F). On treatment day P27, food and water consumption, urine output, and $24 \mathrm{~h}$ urinary albumin excretion were significantly increased in HD compared to sham (Table 1).

OG administrations for 7 days in normal rats did not significantly alter body weight, FBG level, or OGTT values compared with sham-treated rats.

\section{Leukogram}

Alloxan treatment did not alter leukocyte numbers (Figure 2A). In HD rats, the mean percentage of neutrophils was significantly increased (Figure $2 \mathrm{~B}$ ) and the mean percentage of lymphocytes was decreased (Figure 2C); hence, the NLR value was significantly higher in HD compared with sham on P7 and P27 (Figure 2D). The Pearson analysis showed that there were tight correlations between NLR and neutrophils ( $r=0.98, p<0.001)$ (Figure $2 \mathrm{E}$ ) as well as between NLR and lymphocytes $(r=-0.89, p<0.001)$ (Figure $2 F)$. In alloxan-induced DM rats, the same leukogram patterns as in HD rats were found on P27 (Figure $2 \mathrm{G}-\mathrm{I}$ ). With regard to

Table I Kidney weight, kidney index, heart weight, heart index, blood glucose, water intake, food intake, urine output and $24 \mathrm{~h}$ urinary albumin excretion in sham and HD groups on experimental day P27

\begin{tabular}{lll}
\hline Variable & Sham $(\mathbf{n}=8)$ & HD $(\mathbf{n}=7)$ \\
\hline Kidney weight $(\mathrm{g})$ & $1.46 \pm 0.03$ & $1.85 \pm 0.13^{*}$ \\
Kidney index & $6.91 \pm 0.20$ & $9.07 \pm 0.6 \mathrm{I}^{*}$ \\
Heart weight $(\mathrm{g})$ & $0.66 \pm 0.02$ & $0.62 \pm 0.0 \mathrm{I}$ \\
Heart index & $3.10 \pm 0.10$ & $3.06 \pm 0.16$ \\
Blood glucose $(\mathrm{mg} / \mathrm{dL})$ & $75 \pm 2$ & $83 \pm 5$ \\
Water intake $(\mathrm{mL} / \mathrm{day})$ & $28 \pm 3$ & $158 \pm 42^{*}$ \\
Food intake $(\mathrm{g} /$ day) & $15 \pm \mathrm{I}$ & $33 \pm 7^{*}$ \\
Urine volume $(\mathrm{mL} /$ day $)$ & $9 \pm \mathrm{I}$ & $138 \pm 43^{*}$ \\
Urinary albumin $(\mu \mathrm{g} /$ day $)$ & $18.96 \pm 14.52$ & $83.09 \pm 56.23^{*}$ \\
\hline
\end{tabular}

Notes: Values are expressed as mean \pm SEM. Kidney index: ratio of kidney weight/ body weight $(\mathrm{mg} / \mathrm{g})$; heart index: ratio of heart weight/body weight $(\mathrm{mg} / \mathrm{g}) .{ }^{*} p<0.05$ sham vs HD.

Abbreviations: HD, hidden diabetes; P27, posttreatment day 27. monocytes, eosinophils, and basophils, there were no differences between sham and HD groups (Table 2).

In normal rats, the glucose challenges did not alter leukocyte number, but significantly decreased lymphocyte number and percentage value, relatively increased neutrophil percentage value, and led to the increase in the NLR (Figure $3 \mathrm{~A}-\mathrm{F}$ ). Only $1 \mathrm{~h}$ post-glucose administration $(2 \mathrm{~g} / \mathrm{kg})$, lymphocyte percentage values decreased from precontrol $73.88 \pm 13.42 \%$ and $83.42 \pm 5.86 \%$ to $59.80 \pm 14.34 \%$ and $54.32 \pm 11.96 \%$ in normal sham rats and OG-treated rats, respectively $(p<0.05$ and $p<0.01$, respectively). In a comparison of sham with OG treatment, the lymphocyte percentage value dropped further in OG-treated rats at $1 \mathrm{~h}$ after glucose administration $(p<0.05)$. This resulted in a 1.97-fold increase in NLR in sham but a 5.64-fold increase in NLR in OG-treated rats. There was a positive correlation ( $r=0.58, p<0.05$ ) (Figure $3 \mathrm{G}$ ) between the change in blood glucose level and the change in lymphocyte numbers, which resulted from the difference between precontrol and $1 \mathrm{~h}$ after glucose administration. The disorder of innate immune homeostasis caused by OG may be produced by glucose-induced oxidative stress in lymphocytes, as GSH totally blocked the OG-induced alterations in lymphocyte and NLR values (Figure $3 \mathrm{~A}-\mathrm{F}$ ).

\section{Pancreatic and renal histopathology}

Histopathological changes in rats with HD are shown in Figure 4 . The pancreas showed marked $\beta$-cell damage. The islets were more irregular and smaller in alloxan-treated rats than in sham controls. The average islet area was smaller after alloxan treatment (Figure 4A). Compared with sham, the average islet area was 3.7 times smaller $(p<0.001)$ and the islet area to pancreatic area ratio was 5.1 times lower $(p<0.001)$ in HD. Immunohistochemistry studies showed that the staining of islet $\beta$-cells decreased significantly in alloxan-treated animals. On P27, only one-quarter of residual $\beta$-cells remained in alloxan-treated animals $(p<0.001)$ (Figure 4B-D).

Alloxan caused a remarkable change in the kidney weight/ body weight ratio (kidney index) on P27 (Table 1). Histological studies showed that both glomerular areas and volumes were higher in HD than in sham controls on P27 (Figure 4E, F).

\section{Correlations among neutrophils, lymphocytes, NLR, OGTT AUC values, and kidney index}

Correlation analysis showed that both neutrophil and NLR values were positively correlated with OGTT AUC ( $r=0.62$ and $r=0.65$, respectively, both $p<0.01$ ) (Figure $5 \mathrm{~A}, \mathrm{~B}$ ) and 

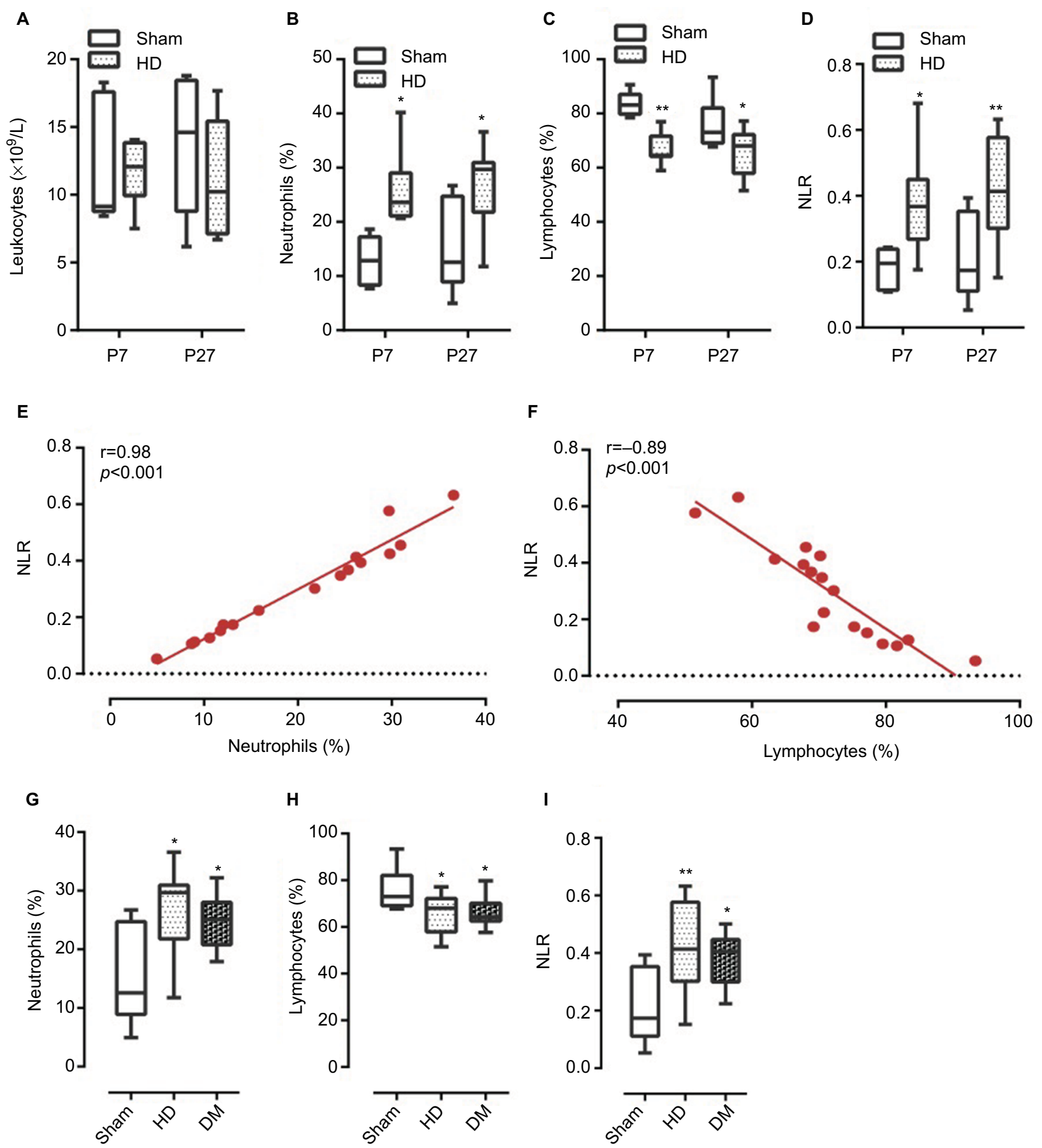

I

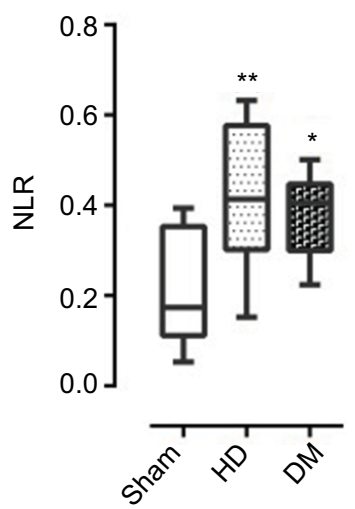

Figure 2 Leukogram of female rats with or without alloxan treatment on P7 ( $n=5$ and $n=6$, respectively) and P27 ( $n=7$ and $n=8$, respectively).

Notes: (A) Leukocyte counts after 7 or 27 days of alloxan treatment. (B) Percentage neutrophils after 7 or 27 days of alloxan treatment. (C) Percentage lymphocytes after 7 or 27 days of alloxan treatment. (D) NLR resulting from (B) and (C). (E) Correlation between percentage neutrophils and NLR on P27. (F) Correlation between percentage lymphocytes and NLR on P27. (G) Percentage neutrophils in sham, HD, and DM groups on P27. (H) Percentage lymphocytes in sham, HD, and DM groups on P27. (I) NLR resulting from $(\mathbf{G})$ and $(\mathbf{H})$. The data from the leukogram were analyzed by analysis of variance with further tests for posthoc comparisons. Relationships between parameters were compared using Pearson correlation analysis. ${ }^{*} p<0.05$ and ${ }^{* *} p<0.01$ sham vs HD.

Abbreviations: P7, posttreatment day 7; P27, posttreatment day 27; NLR, neutrophil-lymphocyte ratio; HD, hidden diabetes; DM, diabetes mellitus. 
Table 2 Leukogram of sham and HD groups on experimental days P7 and P27

\begin{tabular}{lllll}
\hline Variable & P7 & & P27 \\
\cline { 2 - 3 } & Sham (n=6) & HD (n=5) & Sham (n=8) & HD (n=7) \\
\hline Leukocytes ( $\left.\times 10^{9} / L\right)$ & $12.38 \pm 1.52$ & $11.70 \pm 0.85$ & $13.70 \pm 1.56$ & $11.36 \pm 1.49$ \\
Neutrophils (\%) & $12.79 \pm 1.44$ & $25.39 \pm 2.40^{*}$ & $15.08 \pm 2.46$ & $26.68 \pm 2.82^{*}$ \\
Lymphocytes (\%) & $83.36 \pm 1.43$ & $67.83 \pm 2.37 *$ & $76.01 \pm 2.63$ & $65.78 \pm 3.12^{*}$ \\
Monocytes (\%) & $2.55 \pm 0.73$ & $6.52 \pm 1.57$ & $5.63 \pm 1.11$ & $6.73 \pm 2.29$ \\
Eosinophils (\%) & $1.11 \pm 0.25$ & $0.26 \pm 0.13$ & $0.59 \pm 0.30$ & $0.54 \pm 0.32$ \\
Basophils (\%) & $0.17 \pm 0.12$ & $0.00 \pm 0.00$ & $0.00 \pm 0.00$ & $0.00 \pm 0.00$ \\
\hline
\end{tabular}

Notes: Values are expressed as mean \pm SEM. ${ }^{*} p<0.05$ sham vs $H D$.

Abbreviations: HD, hidden diabetes; P7, posttreatment day 7; P27, posttreatment day 27.

kidney index ( $r=0.67$ and $r=0.69$, respectively, both $p<0.01$ ) (Figure 5C, D). In contrast, lymphocyte values were negatively correlated with OGTT AUC $(r=-0.57, p<0.05)$ (Figure $5 \mathrm{E})$ and kidney index $(r=-0.56, p<0.05)$ (Figure 5F). OGTT AUC had a significant tight correlation with kidney index $(r=0.76, p<0.001)$ (Figure 5G).

\section{Discussion}

Rats with HD have usually been considered as unsuccessful candidates for experiments and discarded from studies. Here, in an HD animal model with a restricted and controlled experimental design, our data have shown that alloxan treatment produced $19 \%$ of animals with normal FBG but with abnormal OGTT, diabetic nephropathy, higher neutrophil and lower lymphocyte levels, and a higher NLR. To investigate the mechanism of NLR alteration, we gave rats an acute $2 \mathrm{~g} / \mathrm{kg}$ glucose administration and found that it caused a significant increase in NLR just $1 \mathrm{~h}$ after glucose administration. To our knowledge, this is the first study to report that acute glucose challenge decreased circulating lymphocyte number and percentage, increased the relative percentage of neutrophils, and thus increased the NLR in rats with or without 7 days of OG challenge. The 7 days of OG challenges further potentiated the decrease in the percentage of lymphocytes and resulted in a 5.64-fold increase in the NLR. The disarranging effects of OG and glucose challenge in the homeostasis of the innate immune system were totally blocked by antioxidant GSH administration, suggesting that glucose-induced oxidative stress may play a detrimental role in the maintenance of the homeostasis of innate immune cells. Cells that are exposed to and directly influenced by blood hyperglycemia are blood cells and endothelial cells. Previous studies showed that when endothelial cells were exposed to OG, it was more deleterious than constant high glucose on endothelial function and oxidative stress, two key players in the development of cardiovascular complications in DM. ${ }^{21}$
Many studies on the relationship between NLR and DM have been conducted in patients. ${ }^{22-24}$ Mertoglu and Gunay reported that NLR values were significantly higher in prediabetic, newly diagnosed diabetics by OGTT (HD), and clear diabetic groups compared to a normal group. They suggest that NLR values may be reliable predictive markers in prediabetes and DM. ${ }^{24}$ Here, in an animal HD model, we confirm that NLR was indeed elevated in HD animals. In addition, NLR positively correlated with both the OGTT AUC and the kidney index, suggesting that NLR can be taken as additional information for early diagnosis of $\mathrm{DM}^{25}$ and is also an independent marker for predicting DM, as reported in clinical studies. ${ }^{2}$ It will be possible in the future to make an early diagnosis of $\mathrm{HD}$ when screening DM patients, using a standardized threshold NLR level.

The NLR is widely believed to reflect the inflammatory status in TD1. ${ }^{25}$ The present studies show that the causes of NLR alterations may be produced by the oscillations in glucose levels that are often experienced by diabetic subjects. In HD rats, the residual $\beta$-cells were able to keep the FBG within the normal range. However, the insulin secretion from residual $\beta$-cells was limited and unable to face the OGTT challenge. The animals with abnormal OGTT were unable to maintain glucose homeostasis. The OG challenge may be the cause of NLR changes. Only $1 \mathrm{~h}$ after administration of $2 \mathrm{~g} / \mathrm{kg}$ glucose, although the leukocyte number was not changed the lymphocyte number had decreased significantly, which resulted in the decrease/increase in the percentages of lymphocytes/neutrophils and an increase in the NLR. The antioxidant GSH totally blocked the effects of the OG challenge, suggesting that reactive oxygen species (ROS) play an important role in maintaining the homeostasis of innate immune cells. Consistent with these findings, evidence indicates that both hyperglycemia and alloxan-induced ROS production play a key role in the pathogenesis of diabetic nephropathy. ${ }^{26,27}$ 
A

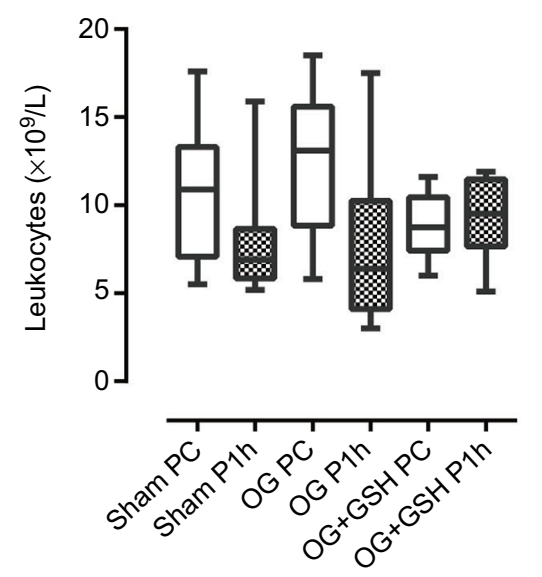

D

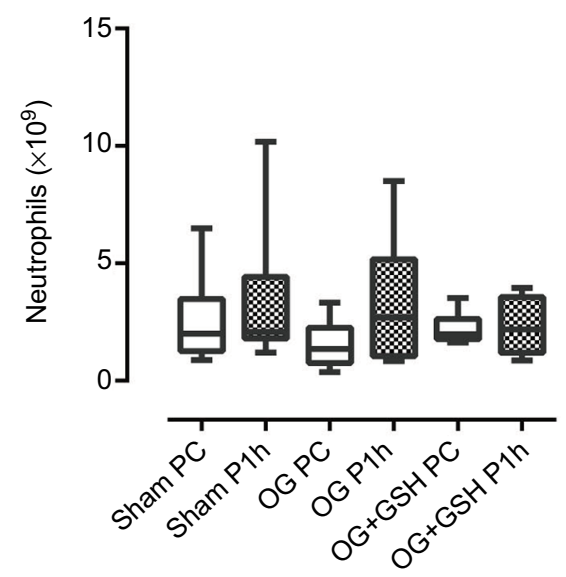

B

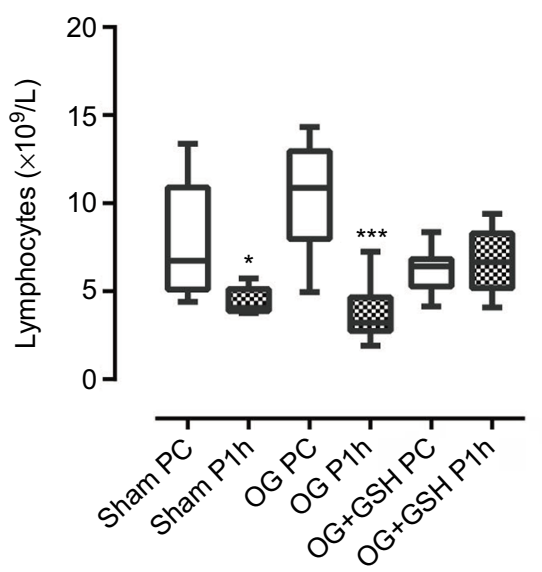

E

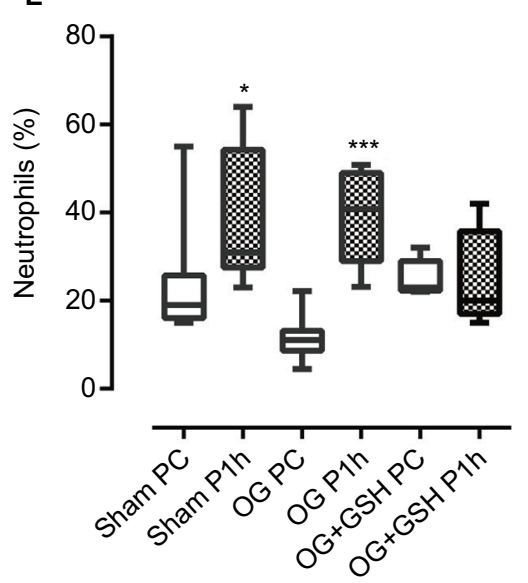

C

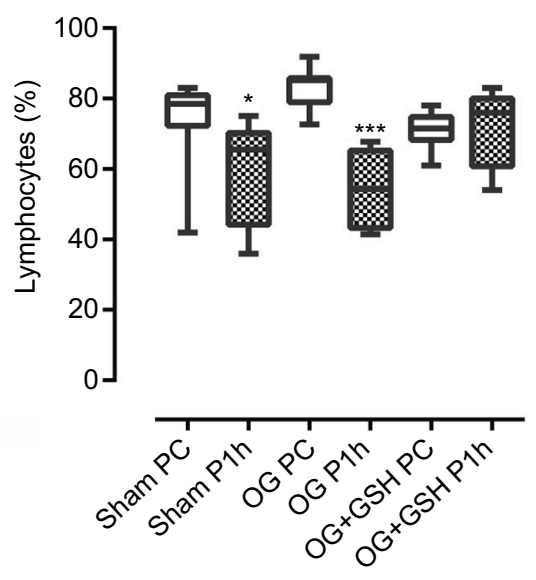

F

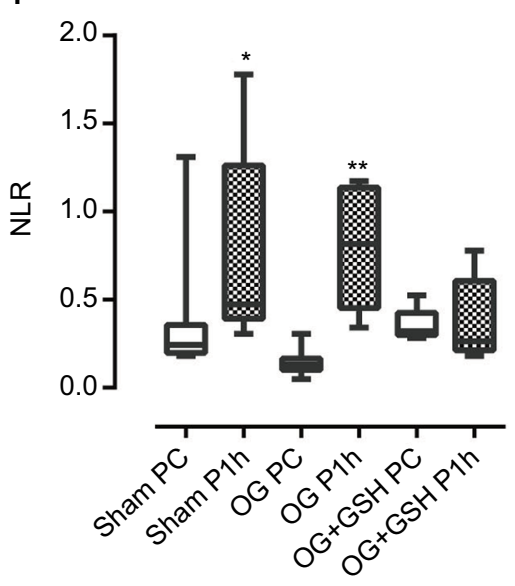

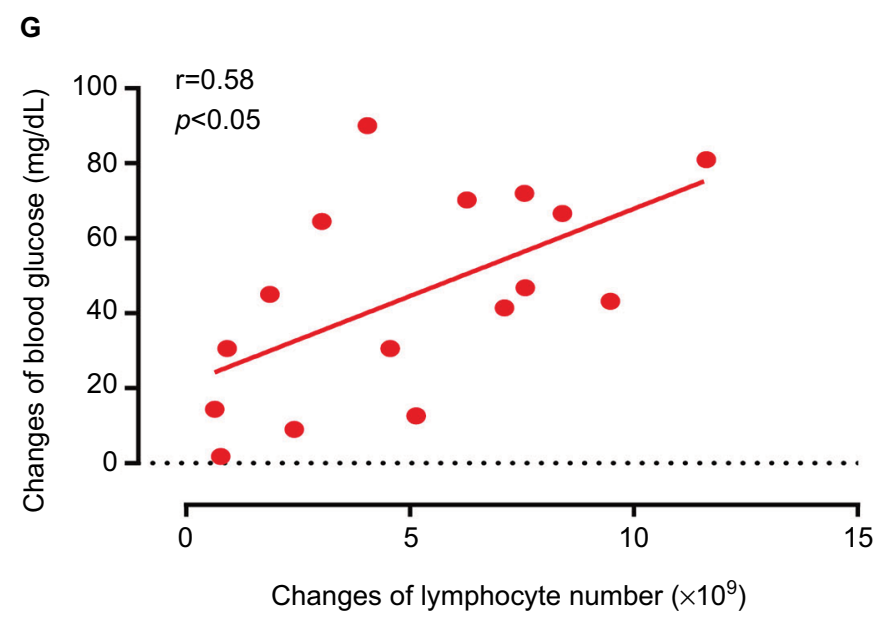

Figure 3 Leukogram of female rats with or without OG and OG+GSH treatments on $\mathrm{P7}$ ( $\mathrm{n}=7$ for all groups).

Notes: (A) Leukocyte counts in PC or PIh treatment. (B) Lymphocyte number after PC or PIh treatment. (C) Percentage lymphocytes after PC or PIh treatment. (D) Neutrophil number in PC or PIh treatment. (E) Percentage neutrophils in PC or PIh treatment. (F) NLR of PC or PIh treatment. (G) Correlation between changes in blood glucose and changes in lymphocyte number. The data from the leukogram were analyzed by 2-way analysis of variance with Tukey tests for posthoc comparisons. Relationships between parameters were compared using Pearson correlation analysis. ${ }^{*} p<0.05$, $* * p<0.01$, and $* * * p<0.00$ I PC vs PIh.

Abbreviations: OG, oscillating glucose; GSH, glutathione; P7, posttreatment day 7; PC, precontrol; PIh, I h post-glucose; NLR, neutrophil-lymphocyte ratio. 

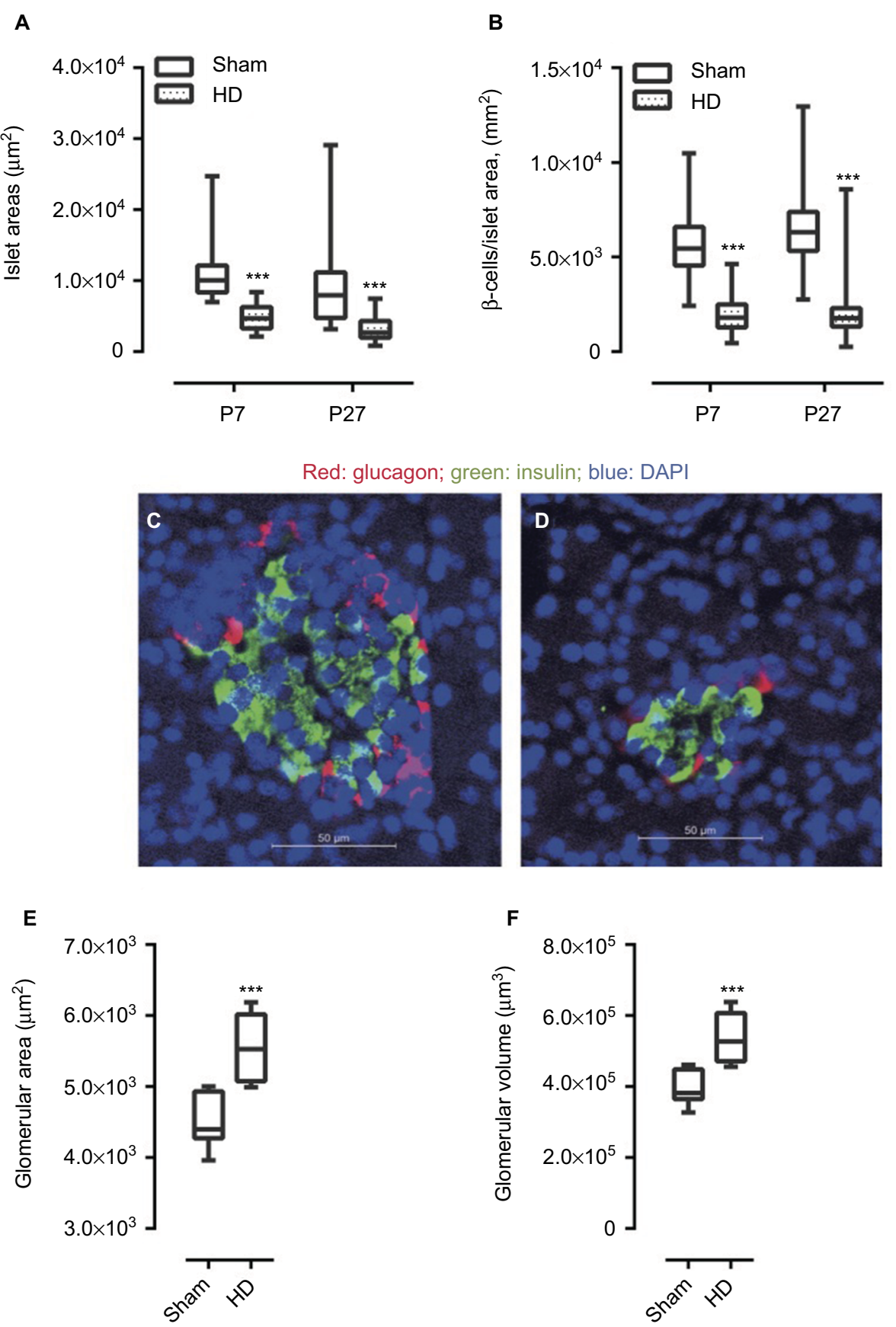

Figure 4 Development of pancreatic and renal pathology of HD with diabetes mellitus on days P7 and P27 after alloxan treatment.

Notes: (A) Islet area (P7: both sham and HD n=18; P27: sham $n=27, H D n=21$ ). (B) $\beta$-cells/islet area ratio (P7: sham $n=173, H D n=84 ; P 27$ : sham $n=135, H D n=58)$. (C) Insulin $+\beta$-cells and glucagon $+\alpha$-cells in sham group on P27 (magnification $\times 400$ ). (D) Insulin $+\beta$-cells and glucagon $+\alpha$-cells in HD group on P27 (magnification $\times 400$ ). (E) Glomerular area (P27: sham $n=1076, \mathrm{HD} n=812$ ). (F) Glomerular volume (P27: sham $n=1076, \mathrm{HD} n=812$ ). *** $p<0.001$ sham vs HD.

Abbreviations: HD, hidden diabetes; P7, posttreatment day 7; P27, posttreatment day 27.

In the alloxan-induced DM model, as the cytotoxic effects of alloxan last for a very short time, ${ }^{28}$ the early nephropathy in alloxan-treated animals may be mainly induced by the alloxan but the late (>21 days) renal disorders would be mainly produced by hyperglycemia. A substantial number of epidemiological studies and animal experiments demonstrate that diabetes is associated with chronic inflammation, which may contribute to the development of DM..$^{29,30}$ The results of the present study suggest that the OG level itself can alter the NLR. Therefore, the increase in neutrophil number is a critical indicator of whether there is an existing inflammatory state. A higher NLR value is usually associated with higher neutrophil numbers and kidney damage. Clinical studies show that patients with an NLR of 1.6-2.4 were almost 
A

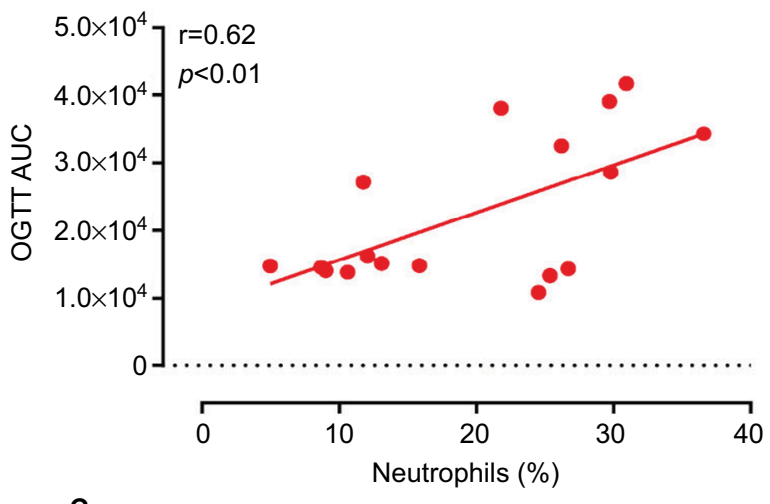

C

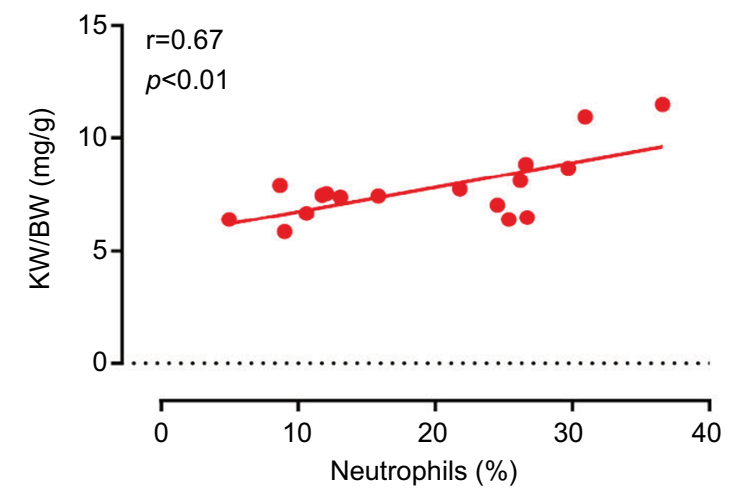

E

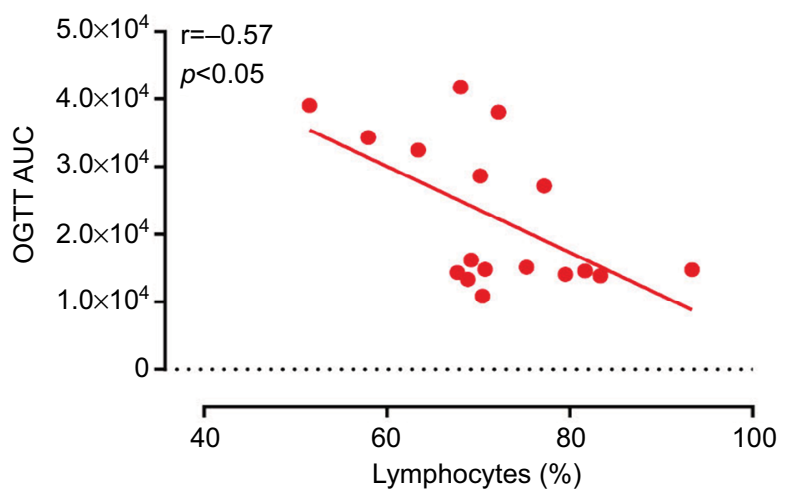

G

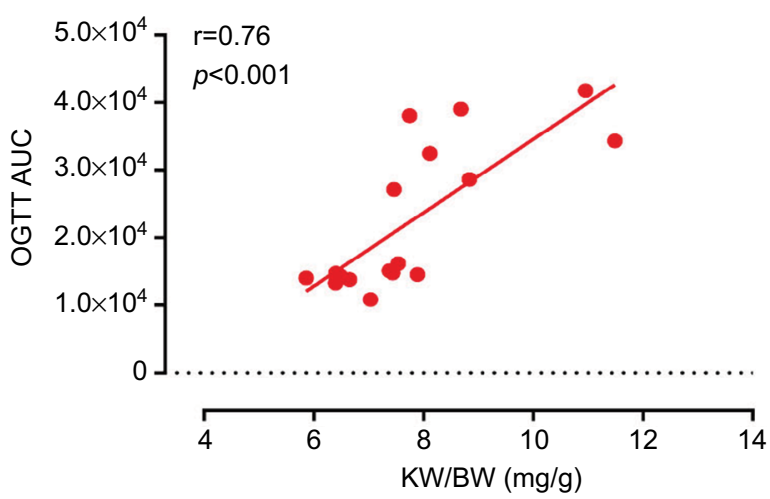

B

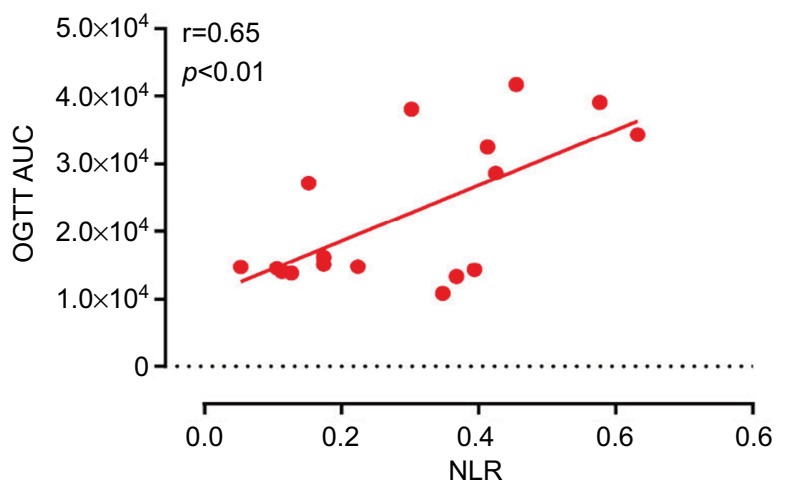

D

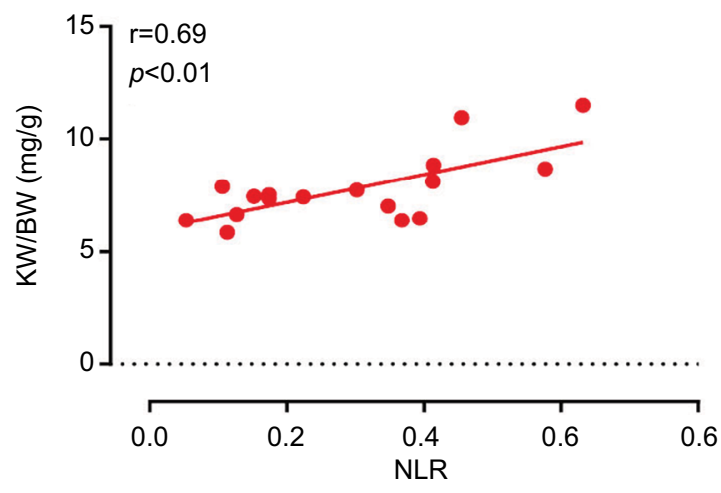

$\mathbf{F}$

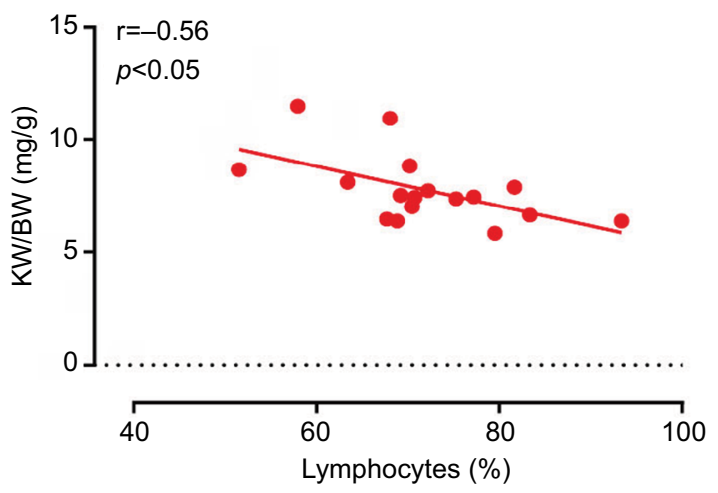

Figure 5 Relationships among leukogram data, glucose metabolic changes, and kidney index values after 27 days of alloxan treatment.

Notes: OGTT AUC values were positively correlated with the percentage of neutrophils (A) and NLR (B). Kidney index values (kidney weight/body weight ratio) were also positively correlated with the percentage neutrophils (C) and NLR (D). The percentage of lymphocytes was negatively correlated with OGTT AUC (E) and kidney index (F). The kidney index values were positively correlated with OGTT AUC (G). Relationships between parameters were compared using Pearson correlation analysis. Abbreviations: OGTT, oral glucose tolerance test; AUC, area under the curve; NLR, neutrophil-lymphocyte ratio; KW/BW, kidney weight/body weight ratio. 
four times more likely to have damaged renal function. ${ }^{23}$ Although we have to be wary in directly applying data from HD animal models to human disease, it is concluded that the use of the alloxan-induced HD rat is suitable for the study of the mechanisms and diagnostic markers contributing to the development and progression of HD and DM.

\section{Conclusion}

Alloxan treatment caused a progressive loss of $\beta$-cell mass in $19 \%$ of female rats with HD. HD associated with elevated NLR values may be partly attributed to a homeostasis disorder of the innate inflammatory state, which was caused by oscillating hyperglycemia. Acute high glucose administration produced a significant decrease in lymphocyte number. OG administration potentiated this effect and increased the NLR value, which was blocked by GSH, suggesting that ROS play a critical role in maintaining lymphocyte numbers. Currently, DM cohorts are mainly screened and judged by the FBG levels, which risks HD being missed. Thus, we recommend that the standard and threshold of NLR value for detecting HD could be clinically established and thus used as a convenient and indicative screening marker.

\section{Acknowledgments}

This work was supported by the National Natural Science Foundation of China (grant 81670336) and the Wenzhou Grant for Scientific Talents (RX2016003), Wenzhou Science and Technology Bureau.

\section{Disclosure}

The authors report no conflicts of interest in this work.

\section{References}

1. Saraheimo M, Teppo AM, Forsblom C, Fagerudd J, Groop PH. Diabetic nephropathy is associated with low-grade inflammation in type 1 diabetic patients. Diabetologia. 2003;46:1402-1407.

2. DiGangi C. Neutrophil-lymphocyte ratio: Predicting cardiovascular and renal complications in patients with diabetes. J Am Assoc Nurse Pract. 2016;28:410-414.

3. Mantovani A, Cassatella MA, Costantini C, Jaillon S. Neutrophils in the activation and regulation of innate and adaptive immunity. Nat Rev Immunol. 2011;11:519-531.

4. Jackson MH, Collier A, Nicoll JJ, et al. Neutrophil count and activation in vascular disease. Scott Med J. 1992;37:41-43.

5. Collier A, Jackson M, Bell D, et al. Neutrophil activation detected by increased neutrophil elastase activity in type 1 (insulin-dependent) diabetes mellitus. Diabetes Res. 1989;10:135-138.

6. Valle A, Giamporcaro GM, Scavini M, et al. Reduction of circulating neutrophils precedes and accompanies type 1 diabetes. Diabetes. 2013;62:2072-2077.

7. Harsunen MH, Puff R, D’Orlando O, et al. Reduced blood leukocyte and neutrophil numbers in the pathogenesis of type 1 diabetes. Horm Metab Res. 2013;45:467-470.
8. Cohen JJ, Duke RC, Fadok VA, Sellins KS. Apoptosis and programmed cell death in immunity. Annu Rev Immunol. 1992;10:267-293.

9. Colucci F, Bergman ML, Penha-Gonçalves C, Cilio CM, Holmberg D. Apoptosis resistance of nonobese diabetic peripheral lymphocytes linked to the Idd5 diabetes susceptibility region. Proc Natl Acad Sci U $S$ A. 1997;94:8670-8674.

10. Jailer JW, Marks DT, Marks PA. Variations in white blood cells following the oral administration of glucose to diabetics and nondiabetics. $J$ Clin Endocrinol Metab. 1948;8:1074-1080.

11. Xu H, Chen Y, Li Y, et al. Mitochondrial apoptosis of lymphocyte is induced in type 2 diabetes. Chin Med J (Engl). 2014;127:213-217.

12. King AJ. The use of animal models in diabetes research. Br J Pharmacol. 2012;166:877-894.

13. Wang J, Wan R, Mo Y, Zhang Q, Sherwood LC, Chien S. Creating a long-term diabetic rabbit model. Exp Diabetes Res. 2010;2010:289614.

14. Federiuk IF, Casey HM, Quinn MJ, Wood MD, Ward WK. Induction of type-1 diabetes mellitus in laboratory rats by use of alloxan: route of administration, pitfalls, and insulin treatment. Comp Med. 2004;54:252-257.

15. Kolb H. Mouse models of insulin dependent diabetes: low-dose streptozocin-induced diabetes and nonobese diabetic (NOD) mice. Diabetes Metab Rev. 1987;3:751-778.

16. Matsumoto K, Ochiai T, Sekita K, Kawasaki Y, Yasuhara K, Furuya T. [Application of an automatic blood cell counter, MICROX, to experimental animals. II. Leukocyte differential count in rabbits, rats, and mice]. Jikken Dobutsu. 1983;32:115-122.

17. Papasouliotis K, Cue S, Crawford E, Pinches M, Dumont M, Burley K. Comparison of white blood cell differential percentages determined by the in-house LaserCyte hematology analyzer and a manual method. Vet Clin Pathol. 2006;35:295-302.

18. Kraynak AR, Storer RD, Jensen RD, et al. Extent and persistence of streptozotocin-induced DNA damage and cell proliferation in rat kidney as determined by in vivo alkaline elution and BrdUrd labeling assays. Toxicol Appl Pharmacol. 1995;135:279-286.

19. Park SK, Kang SK. Renal function and hemodynamic study in obese Zucker rats. Korean J Intern Med. 1995;10:48-53.

20. Li M, Naqvi N, Yahiro E, et al. c-kit is required for cardiomyocyte terminal differentiation. Circ Res. 2008;102:677-685.

21. Ceriello A, Esposito K, Piconi L, et al. Oscillating glucose is more deleterious to endothelial function and oxidative stress than mean glucose in normal and type 2 diabetic patients. Diabetes. 2008;57:1349-1354.

22. Azab B, Chainani V, Shah N, McGinn JT. Neutrophil-lymphocyte ratio as a predictor of major adverse cardiac events among diabetic population: a 4-year follow-up study. Angiology. 2013;64:456-465.

23. Azab B, Daoud J, Naeem FB, et al. Neutrophil-to-lymphocyte ratio as a predictor of worsening renal function in diabetic patients (3-year follow-up study). Ren Fail. 2012;34:571-576.

24. Mertoglu C, Gunay M. Neutrophil-lymphocyte ratio and platelet-lymphocyte ratio as useful predictive markers of prediabetes and diabetes mellitus. Diabetes Metab Syndr. 2016;11 Suppl 1:S127-S131.

25. Huang J, Xiao Y, Xu A, Zhou Z. Neutrophils in type 1 diabetes. $J$ Diabetes Investig. 2016;7:652-663.

26. Winiarska K, Szymanski K, Gorniak P, Dudziak M, Bryla J. Hypoglycaemic, antioxidative and nephroprotective effects of taurine in alloxan diabetic rabbits. Biochimie. 2009;91:261-270.

27. Szkudelski T. The mechanism of alloxan and streptozotocin action in B cells of the rat pancreas. Physiol Res. 2001;50:537-546.

28. Meehan CJ, Davidson PM, Young DG, Foulis AK. The partially diabetic pancreas: a histological study of a new animal model. Pancreas. 1987;2:91-98.

29. Papadopoulou-Marketou N, Chrousos GP, Kanaka-Gantenbein C. Diabetic nephropathy in type 1 diabetes: a review of early natural history, pathogenesis, and diagnosis. Diabetes Metab Res Rev. 2017;33:e2841. Epub 2016 Oct 4.

30. Palsamy P, Subramanian S. Resveratrol protects diabetic kidney by attenuating hyperglycemia-mediated oxidative stress and renal inflammatory cytokines via Nrf2-Keap1 signaling. Biochim Biophys Acta. 2011;1812:719-731. 
Diabetes, Metabolic Syndrome and Obesity: Targets and Therapy is an international, peer-reviewed open-access journal committed to the rapid publication of the latest laboratory and clinical findings in the fields of diabetes, metabolic syndrome and obesity research. Original research, review, case reports, hypothesis formation, expert opinion and commentaries are all considered for publication. The manuscript management system is completely online and includes a very quick and fair peer-review system, which is all easy to use. Visit http://www.dovepress.com/testimonials.php to read real quotes from published authors.

Submit your manuscript here: https://www.dovepress.com/diabetes-metabolic-syndrome-and-obesity-targets-and-therapy-journal 